\title{
NOTE CONCERNING THE NUMBER OF POSSIBLE INTERPRETATIONS OF ANY SYSTEM OF POSTULATES.
}

\author{
BY' PROFESSOR C. J. KEYSER.
}

(Read before the American Mathematical Society December 27, 1917.)

IN this note it is assumed that a mathematical system of postulates contains one or more undefined terms and that at least one of these denotes an element, i. e., a thing as distinguished from a relation. The assignment of an admissible meaning, or value, to each of the undefined elements of a postulate system will be spoken of as an interpretation of the system. By "admissible" meanings are meant meanings that satisfy the postulates or that, in other words, render them true propositions.

A postulate system may be such that from a given interpretation of it a second interpretation may be derived, from the second a third one, from the third a fourth, and so on, in such a way that mathematical induction is available for proving that the system admits of a denumerable infinitude of different interpretations. Such a system, for example, is Hilbert's system for euclidean metric geometry, where the undefined element-names are point, line, and plane. It is well known that one interpretation of this system results from allowing the term point to mean an ordered triad $(x, y, z)$ of real numbers, the term plane to mean the class of triads satisfying an equation of the form $A x+B y+C z+D=0$ where not all the coefficients vanish, and the term line to mean the class of triads satisfying a pair of such equations.

If now we let the equations

$$
\text { (1) } \pi_{1}+\lambda \pi_{2}=0, \quad \pi_{3}+\mu \pi_{4}=0, \quad \pi_{5}+\nu \pi_{6}=0
$$

represent three pencils of planes, where the term plane has the meaning above assigned, it is evident that we may get a second interpretation of the Hilbert system by agreeing that the term point shall mean a triad $(\lambda, \mu, \nu)$ of planes selected as indicated from the given pencils, that by a plane shall be meant a congruence, $a \lambda+b \mu+c \nu+d=0$, of such triads, 
and by a line the one-parameter system of triads common to two such congruences.

To obtain a third interpretation it is sufficient to regard the equations (1) as now representing three pencils of congruences of the foregoing variety and then to take for a point a triad of such congruences, for a plane a congruence of such triads of congruences, and for a line a one-parameter system of them as before.

The process exemplified in obtaining the third interpretation from the second obviously admits of endless repetition, yielding a denumerable infinitude of interpretations of which no two are identical.

The sequence of interpretations thus obtainable, far from including all possible interpretations of the system in question, excludes infinitely many possibilities. One of these is the familiar interpretation which results from agreeing that by a point shall be meant an ordinary point of ordinary inversion space a specified point $O$ of which is treated as non-existent, that a plane shall signify a sphere that would contain $O$ if $O$ were not treated as non-existent, and that a line shall mean the (defective) circle common to two such defective spheres. Starting with this interpretation it would be easy to derive in the way above exemplified an endless sequence of interpretations differing from one another and from those of the foregoing sequence. It is not difficult to see that it would be possible to devise an endless variety of such sequences.

It is noteworthy that in case of any one of the interpretations belonging to either of the two sequences above indicated the ensemble of things denoted by the term plane and the ensemble denoted by the term line are subclasses of the ensemble denoted by the term point; and it is sometimes assumed that this relation of the three terms is required by the Hilbert postulates. That the relation is not required may be shown by an example as follows. In this interpretation the term point is to mean a triad $(\lambda, \mu, \nu)$ of lines selected one from each of the pencils

(2) $L_{1}+\lambda L_{2}=0, \quad L_{3}+\mu L_{4}=0, \quad L_{5}+\nu L_{6}=0$,

which may be in a same plane o: in three different planes; the term plane is to signify a congruence $a \lambda+b \mu+c \nu+d=0$ of conics selected from the conic pencils 
(3) $C_{1}+\lambda C_{2}=0, \quad C_{3}+\mu C_{4}=0, \quad C_{5}+\nu C_{6}=0$,

taken to be in a same plane or in different planes; and the term line is to denote the one-parameter system of quadric surfaces common to a pair of congruences

(4) $\alpha \lambda+\beta \mu+\gamma \nu+\delta=0, \quad \alpha^{\prime} \lambda+\beta^{\prime} \mu+\gamma^{\prime} \nu+\delta^{\prime}=0$

of quadric surfaces selected from, say, three pencils of quadrics

(5) $\quad Q_{1}+\lambda Q_{2}=0, \quad Q_{3}+\mu Q_{4}=0, \quad Q_{5}+\nu Q_{6}=0$.

The questions arise: Does every postulate system admit of an infinite number of interpretations? If so, is the number the same for all systems? If it is, what is the number?

Let $S$ be any postulate system. By hypothesis it contains at least one undefined element-name. Denote it by $e$. Let $I$ be an interpretation of $S$. Suppose the meaning assigned in $I$ to $e$ is $e^{\prime}$. Then all instances, $e_{1}{ }^{\prime}, e_{2}{ }^{\prime}, e_{3}{ }^{\prime}, \cdots$, of $e^{\prime}$ satisfy the postulates of $S$. Let $O$ be any object of thought whatever. This object and any given instance of $e^{\prime}$ together constitute a pair. Let $\epsilon$ be the class of such pairs. Evidently a one-one correspondence subsists between the instances of $e^{\prime}$ and those of $\epsilon$ (the members of the class $\epsilon$ ). If two or more instances, $e_{1}{ }^{\prime}, e_{2}{ }^{\prime}, \cdots$, of $e^{\prime}$ satisfy some relation, we may say that the corresponding instances, $\epsilon_{1}, \epsilon_{2}, \cdots$, of $\epsilon$ also satisfy the same relation, provided we agree to say, as we evidently may, that any instances of $\epsilon$ shall be regarded as satisfying a given relation whenever the $e^{\prime}$-instances contained in the $\epsilon$-instances satisfy the relation. Accordingly, if we assign to $e$ the meaning $\epsilon$ instead of the meaning $e^{\prime}$, we thereby give $S$ a new interpretation $I^{\prime}$. As many different interpretations may be thus obtained as there are objects $O$. In general the difference between two such interpretations will be trivial but it will never be nothing. The conclusion is that any postulate system admits of any given infinite number of interpretations.

Columbia University, December, 1917, 